\title{
Acute myelopathy associated with primary infection with human immunodeficiency virus
}

\author{
DAVID W DENNING, JANE ANDERSON， PETER RUDGE， HILLAS SMITH
}

\section{Abstract}

A 29 year old white homosexual man presented with a two and a half week history of severe sore throat, fever, and extreme fatigue. His symptoms did not respond to antibiotics. He had mild bilateral conjunctivitis, a rash over his chest and back, and enlarged lymph nodes, but examination of the nervous system yielded normal results. He had low total white cell and platelet counts. The results of enzyme linked immunosorbent assay for human immunodeficiency virus (HIV) were equivocal when HIV IgM was detected in serum. Despite treatment with ampicillin his temperature remained high and he developed abnormal neurological signs, including a paraparesis and hyperreflexia of the arms. HIV was isolated from lymphocytes from blood and cerebrospinal fluid. Over the next six weeks the patient improved and was discharged. Two months later abnormal neurological signs persisted in his legs.

Although various neurological syndromes associated with seroconversion to HIV have been described, ${ }^{1-3}$ this is probably the first report of a patient with myelopathy at the time of seroconversion.

\section{Introduction}

Acute myelopathy after primary infection with the human immunodeficiency virus (HIV) has not been reported previously. We report what we believe to be the first case.

\footnotetext{
Departments of Infectious Diseases and Neurology, Northwick Park Hospital and Clinical Research Centre, Harrow, Middlesex HA1 3UJ

DAVID W DENNING, MRCP, DCH, senior registrar

JANE ANDERSON, MB, PHD, senior house officer

PETER RUDGE, BSC, FRCP, consultant neurologist

HILLAS SMITH, MD, FRCP, consultant physician

Correspondence to: Dr Denning.
}

Patient, methods, and results

A 29 year old white homosexual man presented with a two and a half week history of a severe sore throat, a painful mouth, and fever. He developed night sweats, a dry cough, and extreme fatigue and had a constant headache. Before admission he had had a single loose motion after 10 days of constipation. His symptoms did not respond to treatment with penicillin $\mathrm{V}$ or co-trimoxazole. A practising homosexual, he had last had sexual contact about five weeks before the onset of his symptoms. He was taking no prescribed or illegal drugs.

On initial examination his temperature was $38.5^{\circ} \mathrm{C}$. He had mild bilateral conjunctivitis and a rash of discrete red macules over his chest and back. Slightly enlarged lymph nodes were palpable in the neck and left axilla. His buccal mucosa was erythematous with superimposed candida infection, and there was one small flat ulcer. His pharynx was inflamed, but the tonsils were normal and there was no exudate. His liver and spleen were just palpable, and examination of the nervous system yielded normal results.

Investigations showed a low total white cell count of $2 \cdot 2 \times 10^{9} / 1$ and thrombocytopenia (table). Alkaline phosphatase activity was increased at $177 \mathrm{IU} / \mathrm{l}$ and aspartate transaminase activity at $116 \mathrm{IU} / \mathrm{l}$. Results of the Paul-Bunnell test were negative. A throat swab grew Candida albicans. Enzyme linked immunosorbent assay for HIV was equivocal (table).

He received a course of treatment with intravenous ampicillin, but his temperature remained high. On the fifth day after admission he reported that his right leg felt stiff, and his gait became increasingly unsteady. Neurological examination showed an increase in tone in both legs, especially the right, with brisk tendon reflexes and extensor plantar response bilaterally. Power and sensation were normal. Later the same day he suffered an episode of generalised shaking of the legs and experienced increasing difficulty in voiding urine. He seemed mentally slow. Full myelography and computed tomography of the brain yielded normal results. The cerebrospinal fluid contained $7 \times 10^{3} / 1$ lymphocytes (table). No organisms were isolated. Treponema pallidum haemagglutination assay, IgG analysis, and electrophoresis yielded negative results. A working diagnosis of myelitis was made.

Over the next two weeks he continued to experience spasms of uncontrolled shaking of the legs. In addition, he described episodic, severe lancinating pains in his back, which he likened to electric shocks. These improved with clonazepam. He developed a paraparesis, hyperreflexibility of the arms, and a pout reflex. In view of this deterioration a repeat lumbar puncture was performed 10 days after he was admitted.

Blood and cerebrospinal fluid were cultured, and HIV was isolated from a mixture of lymphocytes from both sources (necessitated because of a fungal contamination of the culture of cerebrospinal fluid). Over the ensuing four weeks the patient's condition began to improve, and his gait began to return to normal. His white cell count rose to $6 \cdot 5 \times 10^{9} / 1$ (table). He developed an 
urticarial rash and low grade fever, which was thought to be due to clonazepam and which resolved when chlorpheniramine was given. When discharged he had an increase in tone with normal power in his legs, but his plantar responses had reverted to flexor. Two months later obvious neurological signs persisted in his legs and his walking was still unsteady.

\section{Discussion}

Dementia, myelopathy, and neuropathy in patients with long established HIV infection are well recognised. ${ }^{\prime}$ In the past year neurological syndromes associated with seroconversion to HIV have been described, including examples of patients with meningitis, ${ }^{1}$ encephalopathy, ${ }^{1}$ neuropathy, ${ }^{2}$ and radiculopathy. ${ }^{3}$ Our patient is unique, however, in having a myelopathy at the time of seroconversion. Although the primary site of damage was the spinal immunoassay system. Intrathecal synthesis of HIV specific $\underline{\text { W }}$ immunoglobulin has been shown in asymptomatic carriers of the $?$ virus $^{6}$ and in patients with AIDS. ${ }^{7}$ In one patient with a chronic $\stackrel{\varnothing}{\varrho}$ polyradiculopathy due to HIV sequential analysis showed a cerebro- $C$ spinal fluid:blood IgG synthesis ratio of 14.7 (normal $<1 \cdot 4$ ). ${ }^{7} \widehat{\widehat{O}}$ Unfortunately, the radioimmunoassay system cannot be quantified sufficiently to compute a ratio, and the enzyme linked immuno- $\mathbb{D}^{D}$ sorbent assay is more appropriate to serum (because of high globulin ${ }^{\infty}$ content) than to estimation of cerebrospinal fluid. The antibody detected in the cerebrospinal fluid may therefore represent passive transfer across the blood-brain barrier, although its detection when $\stackrel{5}{9}$ the concentration of antibody in the serum was so low suggestso intrathecal synthesis of antibody to HIV.

The pathogenesis of the acute neurological syndromes is unclear $\overline{\bar{T}}$ in HIV infection. Unlike patients with the chronic syndromes, $\underset{\mathbb{Q}}{\mathscr{2}}$ which are relentlessly progressive, at least some of the patients who

Results of various investigations in patient with HIV infection. Valmes in brackets are actual results of tests



cord, there were subtle signs of dysfunction at a higher level (impaired mental activity and pout reflex); and cortical abnormality was also found in one of the published cases of neuropathy. ${ }^{2}$ At least some of these patients thus seem to have a mild, generalised neurological disturbance, although the clinically significant dysfunction affects a limited part of the central or peripheral nervous system.

The evidence strongly supports a diagnosis of primary HIV infection. One new sexual contact five weeks before the illness would have allowed a typical incubation period. ${ }^{4}$ The other clinical features on admission of fever, rash, enanthem, sore throat, headache, splenomegaly, lympadenopathy, disturbed hepatic function, and leucopenia and thrombocytopenia are characteristic, although not specific. ${ }^{4}$ Oral candidiasis is more typical of the acquired immune deficiency syndrome (AIDS) and suggested a diagnosis of AIDS, although candida may be isolated from the throats of most healthy subjects. The rise in titre of total antibody to HIV, together with the presence of IgM (detected by a modification of the IgG antibody capture system ${ }^{5}$ ) and resolution of the haematological abnormalities, is diagnostic of primary HIV infection. Isolation of the virus adds weight to the serological evidence.

Intrathecal antibody could be detected by a capture radio- present with neurological dysfunction at the time of seroconversion either do not deteriorate or improve, as our patient did. Whether such patients will later develop further neurological disability is unknown.

We thank Dr P Mortimer, Virus Reference Laboratory, Colindale, for performing the HIV serology and Dr A Dalgleish for isolating the virus. We also thank Pauline McAree for typing the manuscript.

\section{References}

1 Black PH. HTLV-III, AIDS and the brain. $N$ Engl $\mathcal{Y}$ Med 1985;313:1538-9.
2 Piette AM, Tusseau F, Vignon D, et al. Acute neuropathy coincident with seroconversion for anti- $\mathrm{N}$

3 Przedborski S, Liesnard C, Hildebrand J. HTLV-III and vacuolar myelopathy. $N$ Engl 7 Med 1986;315:63.

4 Cooper DA, Gold J, MacLean P, et al. Acute AIDS retrovirus infection-definition of a clinical illness associated with seroconversion. Lancet $1985 ; \mathrm{i}: 537-40$.

5 Parry JV. An IgG capture assay (GACRIA) for anti HTLV III/LAV and its use as a confirmatory $\stackrel{\text { + }}{+}$ test. $\mathcal{f}$ Med Virol 1986;14:387-97.

6 Gondsmit J, Wolters EC, Bakker $M$, et al. Intrathecal synthesis of antibodies to HTLV-III in patients without AIDS or AIDS-related complex. Br Med $\mathcal{F}$ 1986;292:1231-4.

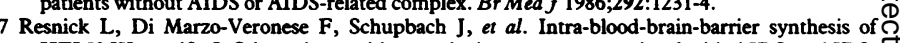
HTLV-III-specific IgG in patients with neurologic symptoms associated with AIDS or AIDS- $\mathbb{D}$ related complex. N Engl I Med 1985;313:1499-504.

(Accepred 28 October 1986) 